\title{
Lower Limb Compression Socks with Biomechanical Concepts
}

\author{
Sergio Daniele ${ }^{1 *}$, Gianluca Rosso ${ }^{3}$, Luca Malfatti ${ }^{3}$, Mauro Testa $^{1}$ and Andrea Licciardi ${ }^{2}$ \\ ${ }^{1}$ Owner Biomoove, Biomechanics Sports Center, Almese, Torino, Italy \\ ${ }^{2}$ Fitness Coach, Torino Football Club, Torino, Italy \\ ${ }^{3}$ Owner, Quant4sport Reaserch, Torino, Italy
}

*Corresponding author: Sergio D, Biomoove, Biomechanics Sports Center, Almese, Torino, Italy

\section{ARTICLE INFO}

Received: 幽 December 15, 2020

Published: 陆 December 22, 2020

Citation: Sergio Daniele, Rosso Gianluca, Malfatti Luca, Testa Mauro and Andrea Licciardi. Lower Limb Compression Socks with Biomechanical Concepts. Biomed J Sci \& Tech Res 32(5)-2020. BJSTR. MS.ID.005301.

Keywords: Compression Garment; Design; Fitness Applications; Model; Pressure; Sports Applications

\section{ABSTRACT}

Graduated compression stockings are now widely used in many sports both for recovery and during the competition with the aim of improving the efficiency of athletes in these two aspects of their sporting life. It does not seem very clear whether the compression of the lower limbs can improve performance, but it does appear evident that it is very useful in the recovery phase. There is also a tendency for compression stocking manufacturers to only offer stockings that begin compression at the ankle continuing upwards, with the primary goal of compressing the calf versus beginning compression at the midfoot upwards. Knowledge of the anatomy and venous biomechanics of the lower limb suggest the presence of an important amount of venous stasis at the level of the foot in correspondence with the midfoot. The benefits of compressing, or "squeezing" this area of the foot using the compression stockings are important in both walking and running, with running reaching a $12 \%$ increase in oxygen supply levels at the femoral area.

For this reason, 14 female runners were recruited for this study, all of them performing the same tests wearing the control stockings, compression stockings that begin compression at the ankle moving upwards, and the stockings with the innovative contribution of the special screen printing of fabrics that begin compression at the midfoot level continuing upwards. Three tests were carried out, the first where the seated runners kept the dominant limb raised to pelvis height, the second where they walked at a speed of $6 \mathrm{~km} / \mathrm{h}$ for 3 minutes with a treadmill inclined at $0.5 \%$, and the third where they were running at $9 \mathrm{Km} / \mathrm{h}$ for two minutes with treadmill inclined at $0.5 \%$. The conclusion of this study from the data collected was that there was an increase in oxygen based on the physical exercise required. In running, the increase in oxygen supply was maximal due to the dynamics of the squeezing forces of the plantar foot using the innovative compression stockings with the special screen printing of fabrics designed to optimize compression.

This study highlights the comparison between two types of compression stockings, one that compresses from the calf upwards, and one that compresses from the midfoot upwards. In summary, the combination of compression stockings with special screen printing of fabrics that compresses from the midfoot upwards improves the efficiency of the venous system during physical activity and is therefore optimal to all sports in both the open and closed kinetic chains. 


\section{Introduction}

The use of compression stockings is increasingly widespread in sports as a post-exercise recovery system or to increase performance. We are confident that the improvement of venous return, "linked to the lymphatic one", can help the elimination of waste metabolites derived from physical exercise or it can bring a greater amount of arterial blood, therefore rich in oxygen, to the muscles to facilitate tissue repair processes and increase performance [1,2]. One of the most effective methods to guarantee venous return is "the pressure gradient". According to this law of physics, liquids and gases go from the point of greatest pressure to that of least. We can understand this concept better by thinking about what happens when we inflate a balloon. The internal pressure will make it fly if we let it vent from the valve because the internal air (higher pressure) will move to where the pressure is lower, (i.e. the air of the environment) [3]. In this way, the greater pressure, adjustable at the level of the plantar arch in standing position, allows the blood to overcome the force of gravity and return to the heart where the pressure is lower.

Venous return is the retrograde flow that brings blood back to the heart. In resting conditions, the venous return must be equal to the cardiac output, because the cardiovascular system is essentially a closed circuit. Otherwise, the blood would accumulate either in the systemic circulation or in the pulmonary circulation. Although cardiac output and venous return are interdependent, each of them can be adjusted independently. The circulatory system is in fact made up of two circulations (pulmonary and systemic) placed in series between the right ventricle (VD) and the left ventricle (VS). The balance is achieved, in large part, by Frank Starling's law. In hemodynamics, the venous return to the heart from the venous vascular bed, located in the midfoot is determined by a pressure gradient (venous pressure right atrial pressure) and by venous resistance: In fact, a decrease in right atrial pressure or venous resistance will lead to an increased venous return, except when changes are determined by posture [4].

From an anatomical point of view, there have been many discussions regarding a structure called Lejiar's insole, positioned in the sole of the foot, attributing it the role of hosting venous stasis. Anatomical studies reveal the existence of the same but attribute it a different role for the venous return more linked to pathological aspects. However, the same studies highlight the importance of compressing the foot in this area as there is an important number of deep veins containing an important amount of venous blood that must be returned to the heart in order to ensure an efficient behavior of the metabolic system connected to the venous return [5]. The venous return and the general well-being of the body that originates from the function of the foot is of primary interest for the biomechanist who is very interested in the prevention of injury during physical activity. We have said that the lymphatic return is closely linked to the venous return. This is the body's "scavenger", that is the system that eliminates waste due to our metabolism or those produced by physical exercise as waste or oxidation elements.

It can therefore be concluded that recovery after a workout or a competition also originates from the foot. Surely in all those sports where we must use the foot its health, even metabolic, guarantees the correct performance. Furthermore, the foot also plays a fundamental role in general well-being. The foot is much more than a simple anatomical structure that allows locomotion. The foot is not limited to supporting the body weight in an upright position. Its vascularization and relative innervation allow it to play an important physiological role, while its osteo-articular structure allows it to adapt to any situation of static or dynamic equilibrium. It can therefore be considered a real organ that actively participates in motor functions. The ability to move body mass is not only linked to the muscle function of the lower limbs and the flexed extensor muscles of the pelvis and knee but also to that of the foot and arch muscles. The foot lever itself is defined as a second order lever and as such is mechanically favorable.

Its ability to push in the sprint phase (for instance), being the fulcrum of the lever, itself positioned at the level of the metatarsal heads, is predominantly horizontal with less vertical component. This mechanical functioning, regulated by the foot musculature, activates the Achilles tendon which stores potential elastic energy in the calf, helping through the contractions of the same, the venous squeezing and consequently there is a facilitation of the venous return itself. These biomechanical aspects related to the movement and function of the foot must be contained in the compression stocking taking them into consideration already starting from its design phase. These considerations do not seem to justify a compression stocking that does not consider the foot but that starts with its squeezing function from the ankle. Evidence of the benefits of compression of the plantar area that contains the deep veins of the foot should prompt the industry to review the design principles of these sports apparel aids.

In the function of the foot, the deflection of the "vault triangle" must also be taken into consideration during the load acceptance phase in walking dynamics or in running, as a pumping factor and therefore favoring venous return. A sock that, in addition to squeezing, supports the structures of the foot in this area is fundamental for the health of the entire human "motor machine" (the body). Even technical aspects related to the construction of the sock itself have been addressed in the literature with the aim of optimizing its functionality [6].

\section{Methods}

With the aim of understanding the advantages of a graduated compression sock with compression at the level of the plantar arch in the midfoot area, we have studied a protocol design capable 
of highlighting any advantages. We start from the consideration that an improvement in blood venous return biomechanics can influence the supply of oxygen in the muscles in a positive way, that is, having an increase in the same in the muscle area. This aspect is essential since cellular respiration and therefore mitochondrial production is supported on oxygen in order to produce ATP with the waste of $\mathrm{Co}_{2}$ [7]. We also know the importance of hemoglobin as buffering to a condition of increased acidity with a decrease in
$\mathrm{PH}$ due to an increase in $\mathrm{CO}_{2}$ in the blood stream [8]. We chose the femoral vein-artery passage area to collect data on the presence of $\mathrm{O}_{2}$ during exercise. Anatomically inferiorly they become popliteal, crossing this area, then tibial to end in the foot area as a deep vein of the foot. Superiorly they cross the pelvic floor and diaphragm to reach the heart. So, an interesting site for our goals related to the proper functioning of the foot in the venous return (Figure 1).
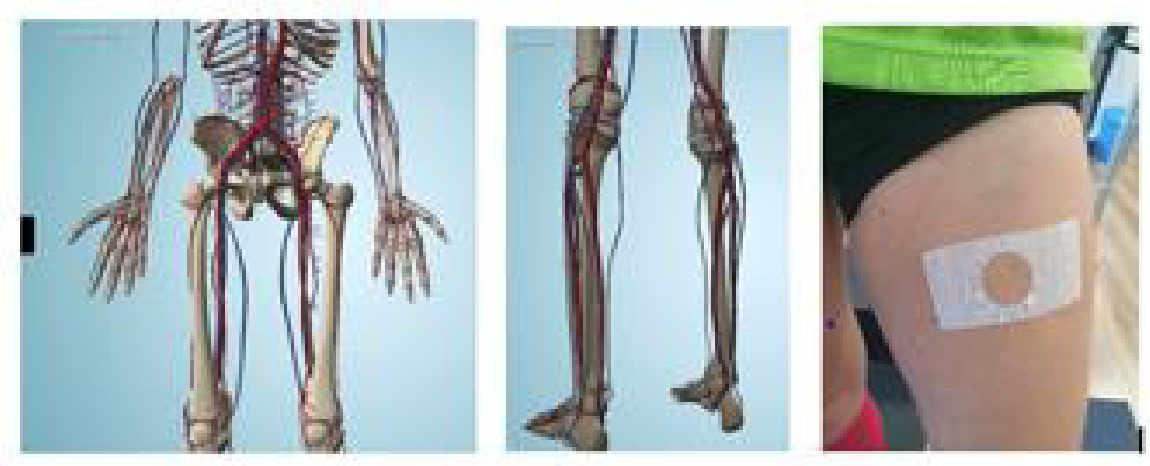

Figure 1: Circulatory System and marker to evaluate venous return.

The running of the athletes (Figure 2), as well as the treadmill walk, took place without the use of footwear to reduce variables and emphasize the functionality of the foot. Although the limits of running on the treadmill are known at a biomechanical level, the same method to reduce the variables is the best to evaluate the functionality of the venous system in the phase of impact with the ground and to consider a similar "squeezing" of the foot for all athletes tested. For the study, 14 semi-professional practicing runners were chosen, each of them first wearing a "control" sock with standard compression, i.e. from the ankle to rising and then the new type of sock with compression in the midfoot. We chose a female population because it would appear from previous studies to be a population more susceptible to getting sick with venous problems $[9,10]$. We defined the dominant limb by asking the subjects to climb over an obstacle and we decided to place the sensor in this part of the lower limbs.

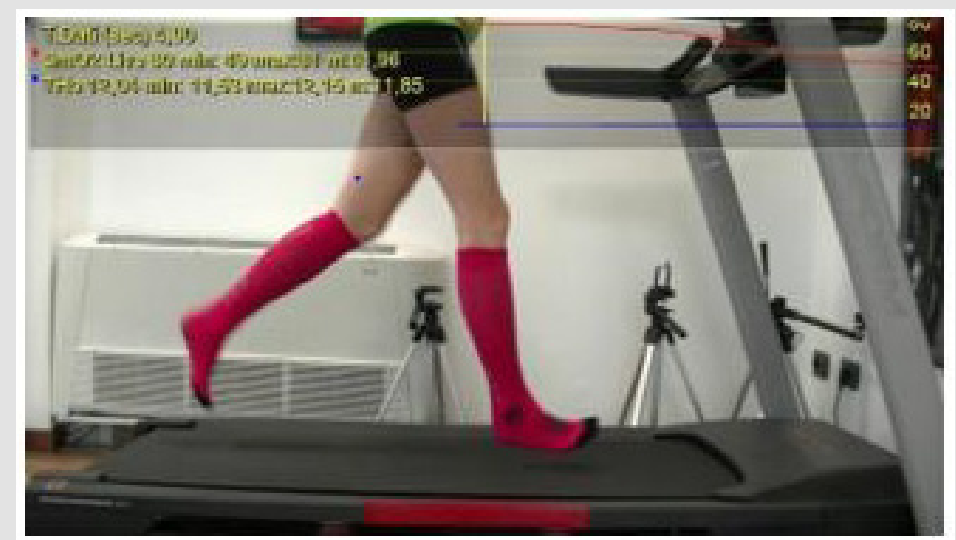

Figure 2: Analysis during running on the treadmill.

We decided to evaluate the subjects in three tests.

1) Simulating the moment of recovery, then with the subject sitting with the dominant limb resting at the height of the pelvis with only the heel touching the support base. Two minutes for this test (Figures 3\&4).

2) Walk on a treadmill at a speed of $6 \mathrm{~km} / \mathrm{h}$ with a belt incline of $0.5^{\circ}$ uphill. Test time 3 minutes Run on the treadmill at a speed of $9 \mathrm{~km} / \mathrm{h}$ with an uphill incline of the belt of $0.5 \%$. Test duration two minutes (Figure 5). The athletes were warmed up before the running and walking test in comparison to the expected physical exertion. Between one test and another we defined recovery times, these recovery times were used to change the socks, so at each test we started with the control sock after which the subject changed the sock putting the one with the compression to the midfoot and then perform the same test. 


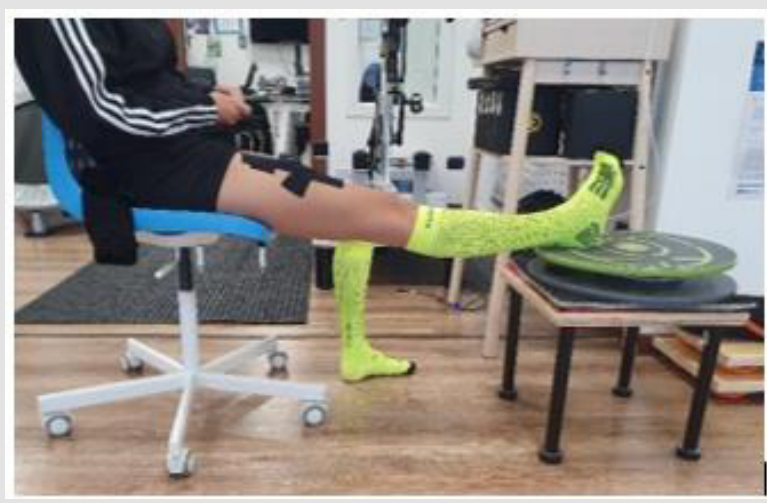

Figure 3: A moment of recovery with RunUp.

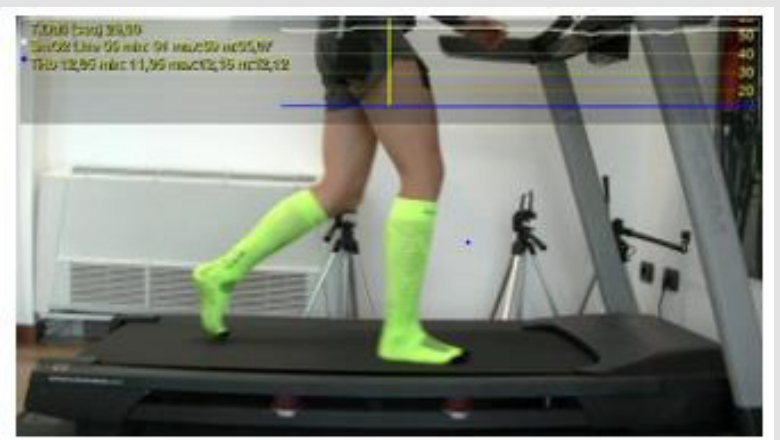

Figure 4: Walk on a treadmill at a speed of $6 \mathrm{~km} / \mathrm{h}$ with RunUp.

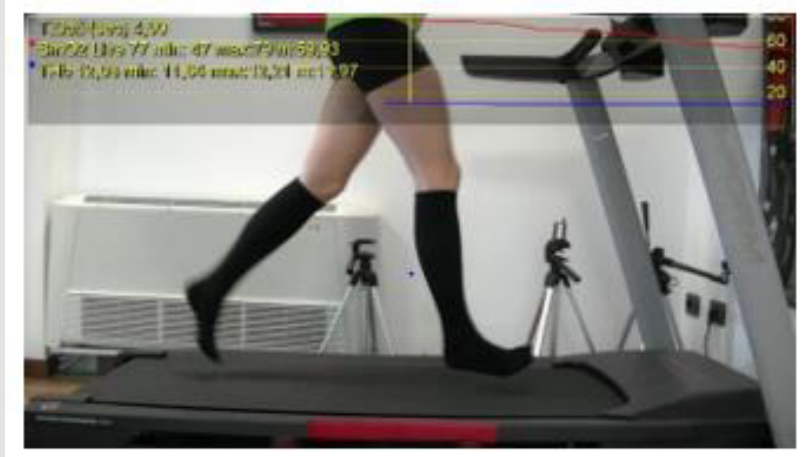

Figure 5: Walk on a treadmill at a speed of $6 \mathrm{~km} / \mathrm{h}$ with RunUp.

All tests were filmed both laterally in the sagittal plane and posteriorly in the frontal plane. The goal of creating movies is to compare the various tests with each other with the aim of verifying their reproducibility. Furthermore, through a video analysis software (Biomovie) we can import the raw data of the sensor into the video by synchronizing them to the movement of the athlete. This allows us to carry out an initial evaluation of the data from a statistical point of view. Additionally, there is the possibility to check the accuracy of the data or any loss of the same every 2 seconds having the data connected to the movement visible in the video and verify the shape of the graph that develops in the test.

\section{Material}

Moxy oxygen monitor to detect $\mathrm{SMO}_{2}$ thus Oxygen muscle saturation in the muscle and THB means Total haemoglobin. Moxy uses light from the near-infrared wavelength spectrum (light from about 670 to $810 \mathrm{~nm}$ ) to measure muscle oxygenation levels in muscle tissue. Human tissue has low optical absorbance of nearinfrared light, so the light can travel to reasonable depths. The near-infrared wavelength range is particularly useful because hemoglobin and myoglobin change color in that range depending on whether they are carrying oxygen. The sensor measures by using near-infrared light through the skin and detecting part of the light after it has travelled into the muscle fibre and returned to the surface and back into the sensor [11,12]. The Moxy is not a pulse oximeter even though it uses the same technology. The main difference is that Moxy measures muscle oxygen saturation $\left(\mathrm{SmO}_{2}\right)$ in capillaries while a pulse oximeter measures hemoglobin oxygen saturation $\left(\mathrm{SpO}_{2}\right)$ in arteries.

This means that a pulse oximeter only demonstrates the saturation of oxygen exiting the lungs, while Moxy monitors the percentage of hemoglobin and myoglobin that carries oxygen. The goal is to have information about the available oxygen or, in other words, to know how our muscle, during workout, are using oxygen efficiently [13]. $\mathrm{SmO}_{2}$ or muscle oxygen saturation is a relationship measure between oxygenated hemoglobin $\left(\mathrm{O}_{2} \mathrm{Hb}\right)$ and deoxygenated hemoglobin (HHb). Using NIRS the Moxy measures in a relative form (not quantifiable) the amount of hemoglobin present in the muscle under the sensor. Specifically, it differentiates between the amount of haemoglobin carrying oxygen and the amount of haemoglobin that is not carrying oxygenation. This results in the following formula: $\mathrm{O}_{2} \mathrm{Hb} / \mathrm{HHb}+\mathrm{O}_{2} \mathrm{Hb}=\mathrm{SmO}_{2}$

$\mathrm{THb}$ is a relative measure (not quantifiable) of the total amount of hemoglobin that is passing underneath the sensor and is therefore the bottom portion of the $\mathrm{SmO}_{2}$ formula above $\mathrm{CO}_{2} \mathrm{Hb}$ $+\mathrm{HHb}$ ). Could be also an indirect indication of the blood flow. The Moxy was solidly fixed using kinesiotape on the athlete's skin at the height of the femoral vein / artery in the dominant limb, allowing it to be used as wearable technology during dynamic walking and running tests. Fourteen pairs of "control" socks with traditional construction, i.e with compression starting from the ankle to rise. Fourteen pairs of stockings model Floky@ RUN UP with a special screen printing on fabrics which gives uniform compression at the level of the midfoot, $21 \mathrm{~mm} \mathrm{hg}$, with decreasing pressure rising towards the popliteal cavity. The design of the sock provides a slight increase in pressure at the calf level.

There are also added silicone-based silkscreen prints on the calf as stabilisation and on the sole of the foot to increase the stabilization of the same. The innovation related to the screen printing of fabrics increase and uniform pressure in plantar area optimizing the compression rate greatly. Nothing was explained 
to the testers about the differences between the control sock and the new one with midfoot compression to avoid any placebo effect. Biomovie, video analysis software with the aim to link row Moxy's data with the video making during the test [14].

\section{Statistical Analysis}

All analyses were conducted using XL Stats (Addinsoft, Paris, France). We made a ANOVA analysis on the datasets, and after this as a validation of the calculated averages and to verify that the increases are significant and due to the specificity of the difference between the two socks, the T Tests for the $\mathrm{SmO}_{2}$ values were performed for the three analysis situations: seated, walking and running. The p-values determined are close to zero so it can be assumed that the probability that the difference in the averages found is due to chance, and not to the sock itself, is close to 0 .

\section{Results}

Collection of SMo2 and THB in the seated test showed the following were "Mid foot" is the new sock with the compression in the mid foot area; We have a $1.3 \%$ increase in $\mathrm{SMO}_{2}$ with the Mid foot sock, while the haemoglobin data has no significant changes. The data collected from the fast-walking test reports the following; We recorded an increase of $9.1 \%$ in oxygen supply (Smo2) with the Mid foot sock and the value for haemoglobin shows no significant changes. The data collected from a running test related the muscle oxygen supply, report the following; The data highlighted in the Running Test shows an increase in oxygen supply of $12 \%$ with the Mid foot sock and with a decrease in haemoglobin of $1.48 \%$. The noted increase in $\mathrm{SMO}_{2}$ between the three tests, increasing as the intensity of the test and the impact forces (therefore squeezing the foot) confirm the reliability of the data. The centroids confirm the analysis contained in the paper regarding an increase in $\mathrm{SmO}_{2}$ and substantial invariance of THb. The dispersion of values is clear, in groups according to the runner.

The trend lines are very similar, with positive slope: if $\mathrm{SmO}_{2}$ increases, THb also increases (Figure 6). The centroids confirm the analysis contained in the paper regarding an increase in $\mathrm{SmO2}$ and substantial invariance of THb. There are some "noise" data in the upper right and left, probably not so influential (the analysis with Box-Wisher Plot does not highlight outliers). The two trend lines are parallel, but the fact that the "run up" line is higher shows an improvement in the values. The lines are negative oriented: if $\mathrm{SmO}_{2}$ increases, $\mathrm{THb}$ decreases. The ANOVA analysis on the datasets indicates a high significance value, so the averages of the individual datasets are distinct from each other (Figure 7). The centroids confirm the analysis contained in the paper regarding the increase in $\mathrm{SmO}_{2}$ and minimum variation in $\mathrm{THb}$. There is no "noise" data. The two trend lines are not parallel: "control" while increasing $\mathrm{SmO2}$ decreases $\mathrm{THb}$, "run up" increases both, $\mathrm{SmO}_{2}$ and $\mathrm{THb}$ (Figure 8).

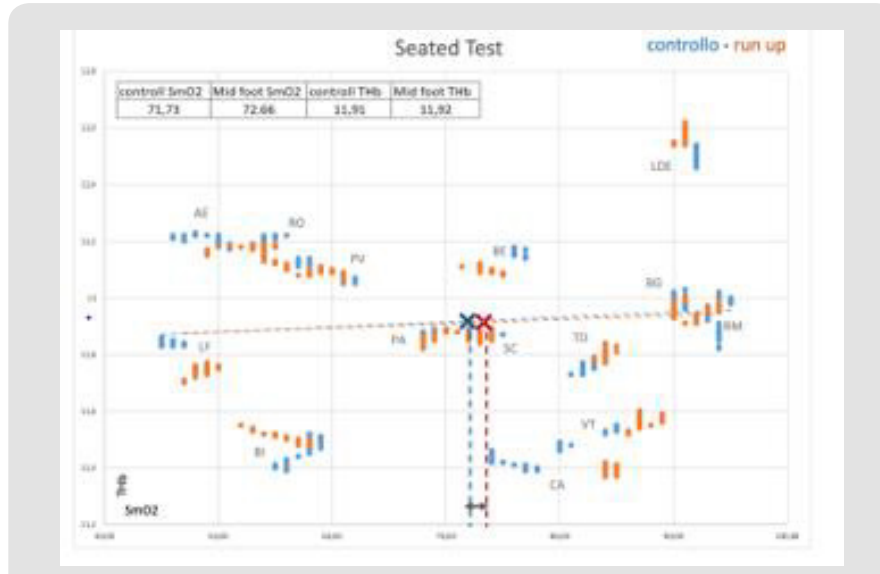

Figure 6: Seated Test.

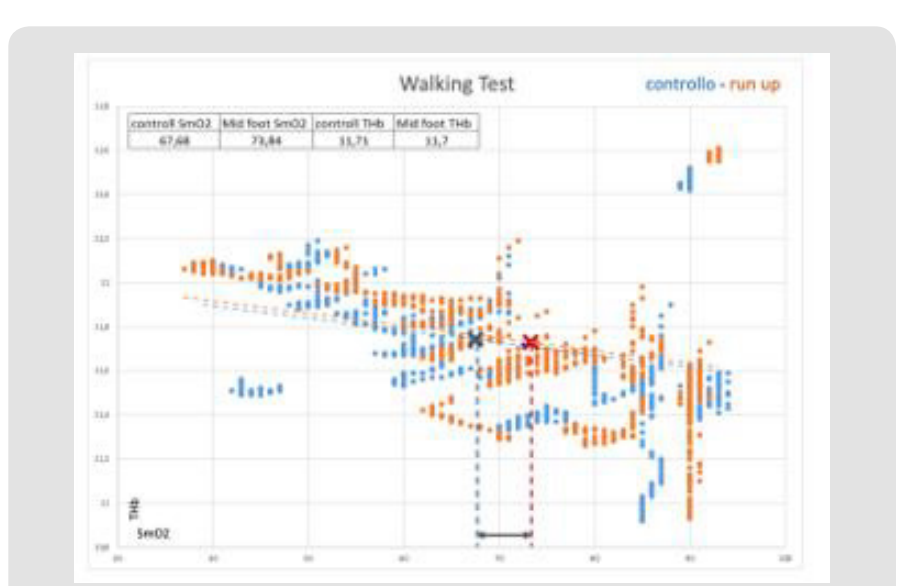

Figure 7: Walking Test.

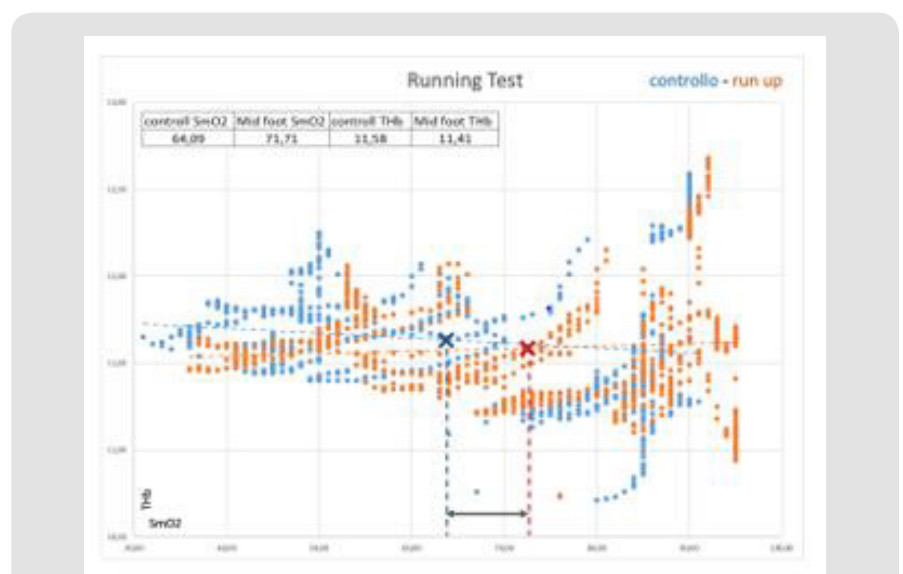

Figure 8: Running Test.

\section{Discussion}

We note an increasing in oxygen in the muscles due to the use of compression stockings starting from the midfoot and this seems to confirm our predictions, in particular we have noticed the importance of the silicon screen printing that wraps the sole of the foot, as well as optimizing the compression avoids any slipping even inside the shoe. In fact, the upward mobilization of blood in 
venous stasis at the level of the sole of the foot increases the volume of blood that reaches the venous part of the heart. An improvement in the "squeezing" of the foot and consequently of the venous return has several consequences including the increase in the circulation of oxygen which also becomes available to the muscle. A better $\mathrm{CO}_{2}-\mathrm{O}_{2}$ balance in the blood stream is also a factor in controlling $\mathrm{pH}$ and therefore acidity $[15,16]$. Oxygen $\left(\mathrm{O}_{2}\right)$ competitively and reversibly binds to hemoglobin, with certain changes within the environment (like CO2 presence) altering the affinity in which this relationship occurs.

The sigmoidal shape of the oxygen dissociation curve illustrates hemoglobin's propensity for positive cooperativity, as hemoglobin undergoes conformational changes to increase its affinity for oxygen as molecules progressively bind to each of its four available binding sites. One of the physiology laws who describe this affinity is the Bohr effect. This principle describes hemoglobin's lower affinity for oxygen secondary to increase in the partial pressure of carbon dioxide and/or decreased blood pH. This lower affinity, in turn, enhances the unloading of oxygen into tissues to meet the oxygen demand of the tissue [17]. Following cellular respiration, which through the presence of $\mathrm{O}_{2}$ and nutrients produces ATP and $\mathrm{CO}_{2}$ as a waste molecule. There is therefore an increase in the circulatory environment of acidosis with a downward alteration of the $\mathrm{PH}$ and this determines, according to what is stated by the effect Bohr, in increased oxygen discharge by haemoglobin [18].

This behaviour of hemoglobin in relation to its affinity with oxygen is called the allosteric effect. During exercise, the muscles then consume oxygen converting it in cellular respiration into ATP and therefore mechanical energy. The $\mathrm{CO}_{2}$ and $\mathrm{H}+$ produced during the breakdown of glucose (nutrients) are removed from the muscle tissues through the blood. The production and removal of $\mathrm{CO}_{2}$ and $\mathrm{H}+$, together with the use and transport of $\mathrm{O}_{2}$, cause chemical changes in the blood. These chemical changes, unless compensated for by other physiological functions, cause the blood $\mathrm{pH}$ to drop. If the body's pH becomes too low (below pH 7.4), a condition known as acidosis occurs. This can be very serious because many of the chemical reactions that occur in the body, especially those involving proteins, are $\mathrm{pH}$ dependent. Ideally, the blood $\mathrm{pH}$ should be kept at 7.4. If the $\mathrm{pH}$ falls below 6.8 or rises above 7.8 , there can be serious consequences for the body and muscle structure with injury or difficulty in recovering.

Fortunately, we have blood buffers to protect against large changes in $\mathrm{pH}$. One of these is hemoglobin itself which can buffer excessive acidity in the blood while maintaining $\mathrm{pH}$ neutrality $[19,20]$. We know that during an exhaustive or high intensity exercise, even if for a short time, the increasing in acidosis and therefore the lowering of the $\mathrm{PH}$ is proportional to it [21].

\section{Conclusion}

The choice of using the foot venous pump in the compression stocking with a screen printing on fabrics in the midfoot like the Floky (C RUN UP, compared to the one commonly used at the ankle/ calf, would seem to have brought benefits in the supply of oxygen and consequently in its availability to the tissues.The improvement in the availability of oxygen in the bloodstream, due to the removal of venous stasis in the podalic area, determines a better balance in the gradients and gas exchanges $\left(\mathrm{O}_{2}-\mathrm{CO}_{2}\right)$ between the tissues, increasing the allosteric effect of hemoglobin upon release of $\mathrm{O}_{2}$. Cellular respiration at the tissue level is consequently able to produce ATP more efficiently and therefore energy currency for movement as well as heat for the body. We hypothesize that the reduction of hemoglobin recorded in the running in the new sock (THB) may be the effect of a lower deoxygenated hemoglobin or a wider use of the same as a buffer. Better management of muscle acidosis with increased $\mathrm{O}_{2}$ availability should promote postworkout recovery, reducing injuries and improving injury healing times.

\section{References}

1. Null M, Agarwal M (2019) Anatomy, Lymphatic System. In StatPearls. StatPearls Publishing.

2. da Silva CA, Helal L, da Silva (2018) Association of lower limb compression garments during high-intensity exercise with performance and physiological responses: a systematic review and meta-analysis. Sports Medicine 48(8): 1859-1873.

3. Brengelmann GL (2019) Venous return and the physical connection between distribution of segmental pressures and volumes.

4. Brengelmann GL (2003) A critical analysis of the view that right atrial pressure determines venous return. Journal of Applied Physiology 94(3): 849-859.

5. Corley GJ, Broderick BJ, Nestor SM, Breen PP, Grace PA, et al. (2010) The anatomy and physiology of the venous foot pump. The Anatomical Record: Advances in Integrative Anatomy and Evolutionary Biology 293(3): 370-378.

6. Gokarneshan N (2017) Design of compression/pressure garments for diversified medical applications. Biomedical Journal of Scientific \& Technical Research 1(3): 806-813.

7. Mairbäurl H (2013) Red blood cells in sports: effects of exercise and training on oxygen supply by red blood cells. Frontiers in physiology 4 : 332.

8. Huang YX, Wu ZJ, Huang BT, Luo M (2013) Pathway and mechanism of pH dependent human hemoglobin tetramer-dimer-monomer dissociations. PLoS One 8(11): e81708.

9. Beebe Dimmer JL, Pfeifer JR, Engle JS, Schottenfeld D (2005) The epidemiology of chronic venous insufficiency and varicose veins. Annals of epidemiology 15(3): 175-184.

10. Lohr JM, Bush RL (2013) Venous disease in women: epidemiology, manifestations, and treatment. Journal of vascular surgery 57(4): $37 \mathrm{~S}-45 \mathrm{~S}$.

11. Crum EM, O'connor WJ, Van Loo L, Valckx M, Stannard SR (2017) Validity and reliability of the Moxy oxygen monitor during incremental cycling exercise. European journal of sport science 17(8): 1037-1043. 
12. Feldmann A, Schmitz RW, Erlacher D (2019) Near-infrared spectroscopyderived muscle oxygen saturation on a $0 \%$ to $100 \%$ scale: reliability and validity of the Moxy Monitor. Journal of biomedical optics 24(11): 115001.

13. McManus CJ, Collison J, Cooper CE (2018) Performance comparison of the MOXY and PortaMon near-infrared spectroscopy muscle oximeters at rest and during exercise. Journal of biomedical optics 23(1): 015007.

14. Souza RB (2016) An evidence-based videotaped running biomechanics analysis. Physical Medicine and Rehabilitation Clinics 27(1): 217-236.

15. Ricci S, Moro L, Antonelli Incalzi R (2014) The foot venous system: anatomy, physiology and relevance to clinical practice. Dermatologic Surgery 40(3): 225-233.

16. Corley GJ, Broderick BJ, Nestor SM, Breen PP, Grace PA, et al. (2010) The anatomy and physiology of the venous foot pump. The Anatomical Record: Advances in Integrative Anatomy and Evolutionary Biology 293(3): 370-378.

\section{ISSN: 2574-1241}

DOI: 10.26717/BJSTR.2020.32.005301

Sergio Daniele. Biomed J Sci \& Tech Res

(C) (i) This work is licensed under Creative

Submission Link: https://biomedres.us/submit-manuscript.php
17. Patel AK, Benner A, Cooper JS (2019) Physiology, Bohr effect. In StatPearls. StatPearls Publishing.

18. Malte H, Lykkeboe G (2018) The Bohr/Haldane effect: a model-based uncovering of the full extent of its impact on $\mathrm{O}_{2}$ delivery to and $\mathrm{CO}_{2}$ removal from tissues. Journal of Applied Physiology 125(3): 916-922.

19. Huang YX, Wu ZJ, Huang BT, Luo M (2013) Pathway and mechanism of pH dependent human hemoglobin tetramer-dimer-monomer dissociations. PLoS One 8(11): e81708.

20. Mairbäurl H (2013) Red blood cells in sports: effects of exercise and training on oxygen supply by red blood cells. Frontiers in physiology 4 : 332 .

21. Junior AHL, de Salles Painelli V, Saunders B, Artioli GG (2015) Nutritional strategies to modulate intracellular and extracellular buffering capacity during high-intensity exercise. Sports Medicine 45(1): 71-81.

$\begin{array}{ll}\text { BIOMEDICAL } & \text { Assets of Publishing with us } \\ \text { RESEARCHES } & \text { - Global archiving of articles } \\ \text { - Immediate, unrestricted online access } & \text { - Rigorous Peer Review Process } \\ & \text { - Authors Retain Copyrights } \\ \end{array}$

